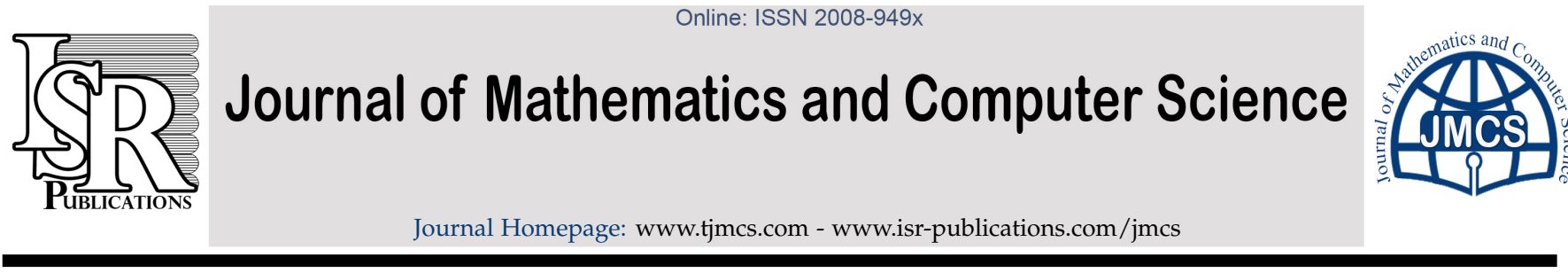

\title{
On the reversible geodesics of a Finsler space with special $(\alpha, \beta)$-metric
}

\author{
Mohammad Rafee $^{\mathrm{a}, *}$, Avdhesh Kumar $^{\mathrm{a}}$, G. C. Chaubey ${ }^{\mathrm{b}}$ \\ ${ }^{a}$ Department of Mathematics, I. K. Gujral Punjab Technical University, Kapurthala, India. \\ ${ }^{b}$ Department of Mathematics, T. D. P. G. College, V. B. S. Purvanchal University, Jaunpur, India.
}

\begin{abstract}
This paper deals with the existence of reversible geodesics on a Finsler space with some $(\alpha, \beta)$-metrics. The conditions for a Finsler space $(M, F)$ to be with reversible geodesics are obtained. We study some geometrical properties of $F$ with reversible geodesics and prove that the Finsler metric $F$ induces a weighted quasi-metric $d_{F}$ on $M$.
\end{abstract}

Keywords: Reversible geodesics, $(\alpha, \beta)$-metric, distance, quasi-metric, weighted quasi-metric.

2010 MSC: 53C60, 53C22.

(C)2018 All rights reserved.

\section{1. Introduction}

The study of Finsler metrics with reversible geodesics is an interesting topic in Finsler geometry. We study the problem with Finsler spaces with $(\alpha, \beta)$-metrics. A Finsler space is said to have reversible geodesics if for every one of its oriented geodesic paths, the same path traversed in the opposite direction is also a geodesic. Some progress has been done in last few years on the study of Finsler spaces with reversible geodesics. In [1], Crampin discusses Randers space with reversible geodesics. In [4, 5], Masca et. al discuss reversible geodesics with $(\alpha, \beta)$-metrics and two dimensional $(\alpha, \beta)$-metrics with reversible geodesic respectively. In [6], Sabau and Shimada discuss Finsler manifolds with reversible geodesics. Motivated by these works, we extend the idea of reversible geodesics to a Finsler space with a special $(\alpha, \beta)$-metric. The paper is organized as follows: In Sect. 2 we discuss some preliminary concepts of Finsler geometry and reversible geodesics. In Sect. 3 we discuss reversible geodesics of a Finsler space with a special $(\alpha, \beta)$-metric. In Sect. 4 we study the relation between Finsler metric and weighted quasimetric.

\section{Preliminaries}

Let $\mathrm{F}^{\mathrm{n}}=(\mathrm{M}, \mathrm{F})$ be an $\mathrm{n}$-dimensional connected, Finsler manifold equipped with a Finsler metric $\mathrm{F}$. $\mathrm{F}: \mathrm{TM} \rightarrow[0, \infty)$ is a Finsler norm, i.e., smooth function on $\widetilde{\mathrm{TM}}=\mathrm{TM} \backslash\{0\}$, with the homogeneous property $F(x, \lambda y)=\lambda F(x, y)$ for all $\lambda>0$ and all $(x, y) \in \widetilde{T M}$ and whose Hessian matrix $\left(g_{i j}\right)=\left(\frac{1}{2} \frac{\partial^{2} F^{2}}{\partial y^{i} \partial y^{j}}\right)$ is positive

\footnotetext{
*Corresponding author

Email address: mohd_rafee60@yahoo.com (Mohammad Rafee)

doi: $10.22436 /$ jmcs.018.03.12

Received: 2018-05-28 Revised: 2018-07-04 Accepted: 2018-08-02
} 
definite at each point $u=(x, y) \in \widetilde{T M}$. If $\gamma:[0,1] \rightarrow M$ is a piecewise $C^{\infty}$ curve on $M$, then its Finslerian length of $\gamma$ is defined as [7],

$$
\mathrm{E}_{\mathrm{F}(\gamma)}=\int_{0}^{1} \mathrm{~F}(\gamma(\mathrm{t}), \dot{\gamma}(\mathrm{t})) \mathrm{dt}
$$

In order for this expression to be invariant under reparameterizations of curve $\gamma$, a requirement on $F$ is imposed, that is, $F(x, \lambda y)=\lambda F(x, y)$ for all $\lambda>0$, in the definition of Finsler metric. Length structures obtained from this type of constructions are called Finslerian length structure. Now, since we have a length structure therefore we can measure any distance on the manifold $M$. Consequently, this length structure induces a distance function $d_{F}: M \times M \rightarrow[0, \infty)$, known as Finslerian distance function, that is defined by $d_{F}(p, q)=i n f_{\gamma} L$, where infimum is taken over all piecewise $C^{\infty}$-curves joining the points $p=\gamma(0)$ to $q=\gamma(1)$. In general, Finslerian distance function $d_{F}$ is irreversible, i.e., $d_{F}(p, q) \neq d_{F}(q, p)$.

If $(M, g)$ is a Riemannian manifold, where $g$ is Riemannian metric, then it is easy to check that its geodesics are reversible, i.e., if $\gamma:[0,1] \rightarrow M$ is a geodesic of $g$, then $\bar{\gamma}:[0,1] \rightarrow M, \bar{\gamma}=\gamma(1-t)$ is also a geodesic of $\mathrm{g}$. In case of an arbitrary Finsler manifold $(M, F)$, this property is not always true. However, there are some special Finsler structures on smooth manifold whose geodesics are reversible. Our main aim is to characterize such Finsler metrics which play a role in order to have a reversible geodesics.

A curve $\gamma:[0,1] \rightarrow M$ is called geodesic of Finsler manifold $(M, F)$ if it minimizes the Finslerian length defined by Equation (2.1) for all piecewise $C^{\infty}$-curves that keep their endpoints fixed. Denote the reversible Finsler metric of $F$ as a smooth function $\bar{F}: T M \rightarrow[0, \infty)$, given by $\bar{F}(x, y)=F(x,-y)$. It is easy to see that $\bar{F}$ is also a Finsler metric. Recall the following definition and lemmas $([1,4,6])$ for later use.

Lemma 2.1. If $\gamma(\mathrm{t})$ is a geodesic of the Finsler space $(\mathrm{M}, \mathrm{F})$, then $\bar{\gamma}(\mathrm{t})=\gamma(1-\mathrm{t})$ is a geodesic of $\overline{\mathrm{F}}$, but not necessarily a geodesic of $F$ in general.

Definition 2.2. The Finsler metric $F$ is called with reversible geodesics if and only if for any geodesic $\gamma(t)$ of $\mathrm{F}$, the reverse curve $\bar{\gamma}(\mathrm{t})=\gamma(1-\mathrm{t})$ is also a geodesic of $\mathrm{F}$.

Lemma 2.3. Let $(\mathrm{M}, \mathrm{F})$ be a connected, complete Finsler manifold with associated distance function $\mathrm{d}_{\mathrm{F}}: \mathrm{M} \times$ $\mathrm{M} \rightarrow[0, \infty)$. Then $\mathrm{d}_{\mathrm{F}}$ is symmetric distance function on $\mathrm{M} \times \mathrm{M}$ if and only if $\mathrm{F}$ is absolute homogeneous, i.e., $F(x, y)=\bar{F}(x, y)=F(x,-y)$.

Definition 2.4. A special vector field $\Gamma$, on the slit tangent bundle $\widetilde{T M}=T M \backslash\{0\}$ is said to be spray on smooth manifold $M$ if $\Gamma$ satisfies following properties:

(a) In standard coordinate system $(x, y)$ on $T M, \Gamma$ can be expressed as $\Gamma=y^{i} \frac{\partial}{\partial x^{i}}-2 G^{i}(x, y) \frac{\partial}{\partial y^{i}}$, where $\mathrm{G}^{i}(x, \lambda y)=\lambda^{2} \mathrm{G}^{i}(x, y), \forall>0 . \mathrm{G}^{i}$ are called spray coefficients;

(b) $\mathrm{G}^{i}$ are smooth at $(x, y \neq 0) \in \widetilde{\mathrm{TM}}$.

Spray is sometimes also denoted by $\mathrm{G}$ instead of $\Gamma$.

Locally, a smooth curve $\gamma:[0,1] \rightarrow M$ is a constant Finslerian speed geodesic of $(M, F)$ if and only if it satisfies the system of differential equations $\ddot{\gamma}^{i}(t)+2 G^{i}(\gamma(t), \dot{\gamma}(t))=0, i=1,2, \cdots, n$, where $\dot{\gamma}=\dot{\gamma}^{i} \frac{\partial}{\partial x^{i}}$. Here the functions $\mathrm{G}^{i}: \widetilde{\mathrm{TM}} \rightarrow \mathrm{R}$ are given by

$$
G^{i}(x, y)=\Gamma_{j k}^{i} y^{j} y^{k}
$$

where $\Gamma_{j k}^{i}(x, y):=\frac{1}{2} g^{i s}\left(\frac{\partial g_{s j}}{\partial x^{k}}+\frac{\partial g_{s k}}{\partial x^{i}}-\frac{\partial g_{i k}}{\partial x^{s}}\right)$. The spray $\Gamma:=y^{i} \frac{\partial}{\partial x^{i}}-2 G^{i} \frac{\partial}{\partial y^{i}}$ is a well defined vector field on the slit tangent bundle $\widetilde{T M}=T M \backslash\{0\}$, whose integral lines are canonical lift $\bar{\gamma}(\mathrm{t})=(\gamma(\mathrm{t}), \dot{\gamma}(\mathrm{t}))$ of the geodesics of $\gamma$. Due to this reason, the vector field $\Gamma$ is called the canonical geodesic spray of the Finsler space $(M, F)$ and $G^{i}$ are called the coefficient of the geodesic spray $\Gamma$.

Recall $([1,4])$ for the later use, that the Euler-Lagrange equation of $(M, F)$, in terms of geodesic spray $\Gamma$, is given by $\Gamma\left(\frac{\partial F}{\partial y^{i}}\right)-\frac{\partial F}{\partial x^{i}}=0$. 
Definition 2.5 ([6]). If $\mathrm{F}$ and $\overline{\mathrm{F}}$ are two different Finsler functions on same differentiable manifold M. Then $\mathrm{F}$ and $\overline{\mathrm{F}}$ are called projectively equivalent, or projectively related, if their geodesics coincide as a set of points.

Using geodesics sprays, the above definitions is equivalent to $\bar{\Gamma}\left(\frac{\partial F}{\partial y^{i}}\right)-\frac{\partial F}{\partial x^{i}}=0$, where $\bar{\Gamma}$ is geodesic spray of $(M, \bar{F})$.

Lemma 2.6 ([6]). A Finsler structure (M, F) is with reversible geodesics if and only if $F$ and its reversible function $\overline{\mathrm{F}}$ are projectively equivalent, i.e., the geodesics of $F$ and $\overline{\mathrm{F}}$ coincides as a set of points.

If we denote the reverse geodesic spray by $\bar{\Gamma}$, then $\mathrm{F}$ is with reversible geodesics if and only if $\bar{\Gamma}\left(\frac{\partial F}{\partial y^{i}}\right)-$ $\frac{\partial F}{\partial x^{i}}=0$.

\section{Reversible geodesics of a Finsler space with a special $(\alpha, \beta)$-metric}

Consider a Finsler space $(M, F)$ with a special $(\alpha, \beta)$-metric $F=\alpha+\beta+\frac{\beta^{2}}{\alpha}$, known as first approximate Matsumoto metric. $F$ can be treated as the Randers change of a special $(\alpha, \beta)$-metric $\bar{F}=\alpha+\frac{\beta^{2}}{\alpha}$. One can easily see that $\overline{\mathrm{F}}$ is absolute homogeneous as $\overline{\mathrm{F}}(x,-y)=\overline{\mathrm{F}}(x, y)$.

It is a well known fact [6] that if $(M, F)$ is a non-Riemannian $n(n \geqslant 2)$-dimensional Finsler structure with $(\alpha, \beta)$-metric, which is not absolute homogeneous, then $\mathrm{F}$ is with reversible geodesics if and only if $F(\alpha, \beta)=F_{0}(\alpha, \beta)+\epsilon \beta$, where $F_{0}$ is absolute homogeneous $(\alpha, \beta)$-metric, $\epsilon$ is a non-zero constant and $\beta$ is a closed 1-form on the manifold $M$.

In our case, $F_{0}=\bar{F}$ is absolute homogeneous. If $\beta$ is a closed 1-form, then $F$ is with reversible geodesics. A necessary and sufficient condition for $F$ to have reversible geodesics is that [6]

$$
\bar{\Gamma}\left(\frac{\partial F}{\partial y^{i}}\right)-\frac{\partial F}{\partial x^{i}}=0
$$

where $\bar{\Gamma}$ is the reverse of $\Gamma$, the geodesic spray of $F$; moreover $\bar{\Gamma}$ is geodesic spray of $\bar{F}$. We have

$$
\mathrm{F}=\alpha+\beta+\frac{\beta^{2}}{\alpha}, \overline{\mathrm{F}}=\alpha+\frac{\beta^{2}}{\alpha}
$$

Therefore $F=\bar{F}+\beta$. We have

$$
\begin{aligned}
\bar{\Gamma}\left(\frac{\partial F}{\partial y^{i}}\right)-\frac{\partial F}{\partial x^{i}} & =\bar{\Gamma}\left(F_{\alpha} \frac{\partial \alpha}{\partial y^{i}}+F_{\beta} \frac{\partial \beta}{\partial y^{i}}\right)-F_{\alpha} \frac{\partial \alpha}{\partial x^{i}}-F_{\beta} \frac{\partial \beta}{\partial x^{i}} \\
& =\bar{\Gamma}\left(F_{\alpha}\right) \frac{\partial \alpha}{\partial y^{i}}+F_{\alpha} \bar{\Gamma}\left(\frac{\partial \alpha}{\partial y^{i}}\right)+\bar{\Gamma}\left(F_{\beta}\right)\left(\frac{\partial \beta}{\partial y^{i}}\right)+F_{\beta} \bar{\Gamma}\left(\frac{\partial \beta}{\partial y^{i}}\right)-F_{\alpha} \frac{\partial \alpha}{\partial x^{i}}-F_{\beta} \frac{\partial \beta}{\partial x^{i}} \\
& =\bar{\Gamma}\left(F_{\alpha}\right) \frac{\partial \alpha}{\partial y^{i}}+F_{\alpha}\left[\bar{\Gamma}\left(\frac{\partial \alpha}{\partial y^{i}}\right)-\frac{\partial \alpha}{\partial x^{i}}\right]+\bar{\Gamma}\left(F_{\beta}\right) \frac{\partial \beta}{\partial y^{i}}+F_{\beta}\left[\bar{\Gamma}\left(\frac{\partial \beta}{\partial y^{i}}\right)-\frac{\partial \beta}{\partial x^{i}}\right] .
\end{aligned}
$$

For the Riemannian metric $\alpha$, the Euler-Lagrange equation gives $\bar{\Gamma}\left(\frac{\partial \alpha}{\partial y^{i}}\right)-\frac{\partial \alpha}{\partial x^{i}}=0$. Also, one knows (see lemma 2.7 of [6]) that if $(M, F(\alpha, \beta))$ be a Finsler space with $(\alpha, \beta)$-metric, then $f(x, y) \frac{\partial \alpha}{\partial y^{i}}+g(x, y) b_{i}=0$, $\forall=1,2, \ldots, \mathrm{n}$., implies that $f=g=0$, for any smooth functions $f$ and $g$ on TM. If $\beta$ is closed and $F$ is projectively equivalent to the Riemannian metric $\alpha$, then $\bar{\Gamma}\left(\mathrm{F}_{\alpha}\right) \frac{\partial \alpha}{\partial y^{i}}+\bar{\Gamma}\left(\mathrm{F}_{\beta}\right) b_{i}=0$ and by the lemma 2.7 of [6], we find that $\bar{\Gamma}\left(F_{\alpha}\right)=0, \bar{\Gamma}\left(F_{\beta}\right)=0$. Again, since $F=\alpha+\beta+\frac{\beta^{2}}{\alpha}$, therefore $F_{\beta}=1+\frac{2 \beta}{\alpha}$.

Now, using above results, the Equation (3.2) reduces to the form

$$
\bar{\Gamma}\left(\frac{\partial F}{\partial y^{i}}\right)-\frac{\partial F}{\partial x^{i}}=F_{\beta}\left[\bar{\Gamma}\left(\frac{\partial \beta}{\partial y^{i}}\right)-\frac{\partial \beta}{\partial x^{i}}\right]
$$




$$
\begin{aligned}
& =\left(1+\frac{2 \beta}{\alpha}\right)\left[\bar{\Gamma}\left(\frac{\partial \beta}{\partial y^{i}}\right)-\frac{\partial \beta}{\partial x^{i}}\right] \\
& =\left(1+\frac{2 \beta}{\alpha}\right)\left(\bar{\Gamma}\left(b_{i}\right)-\frac{\partial b_{j}}{\partial x_{i}} y^{j}\right) \\
& =\left(1+\frac{2 \beta}{\alpha}\right)\left(\frac{\partial b_{i}}{\partial x^{j}} y^{j}-\frac{\partial b_{j}}{\partial x^{i}} y^{j}\right) \\
& =\left(1+\frac{2 \beta}{\alpha}\right)\left(\frac{\partial b_{i}}{\partial x^{j}}-\frac{\partial b_{j}}{\partial x^{i}}\right) y^{j} .
\end{aligned}
$$

Here $1+\frac{2 \beta}{\alpha}=0$ is not admissible, so $F$ is with reversible geodesics if and only if $\bar{\Gamma}\left(\frac{\partial F}{\partial y^{i}}\right)-\frac{\partial F}{\partial x^{i}}=0$, which implies that $F$ is with reversible geodesics if and only if $\left(\frac{\partial b_{i}}{\partial x^{j}}-\frac{\partial b_{j}}{\partial x^{i}}\right) y^{j}=0$, i.e., $F$ is with reversible geodesics if and only if $\beta$ is closed 1-form. Hence, we have the following theorem:

Theorem 3.1. A Finsler space (M, F) with first approximate Matsumoto metric $F=\alpha+\beta+\frac{\beta^{2}}{\alpha}$ is with reversible geodesics if and only if the differential 1-form $\beta$ is closed on $M$.

Next, a Finsler space $(M, F)$ is called (locally) projective flat if all its geodesics are straight lines. An equivalent condition is that the spray coefficient $G^{i}$ of $F$ can be expressed as $G^{i}=P(x, y) y^{i}$, where $P(x, y)=\frac{1}{2 F} \frac{\partial F}{\partial x^{k}} y^{k}$.

An equivalent characterization of projective flatness is the Hamel's relation [2]

$$
\frac{\partial^{2} F}{\partial x^{m} \partial y^{k}} y^{m}-\frac{\partial F}{\partial x^{k}}=0
$$

Recall (see theorem 3.1 of [6]) that if $F=F_{0}+\epsilon \beta$ is a Finsler metric, where $F_{0}$ is an absolute homogeneous $(\alpha, \beta)$-metric, then any two of the following properties imply the third one:

(1) $\mathrm{F}$ is projectively flat;

(2) $F_{0}$ is projectively flat;

(3) $\beta$ is closed.

In our case $F=\alpha+\beta+\frac{\beta^{2}}{\alpha}=\bar{F}+\beta$, where $\bar{F}=\alpha+\frac{\beta^{2}}{\alpha}$ which is absolute homogeneous.

Theorem 3.2. Let $(M, F)$ be a Finsler space with first approximate Matsumoto metric. Then $F$ is projectively flat if and only if $\overline{\mathrm{F}}$ is projectively flat.

Proof. Now, first we suppose $F$ is projectively flat. Since F is projectively flat, therefore $F$ will satisfy Hamel's equation

$$
\begin{aligned}
& \frac{\partial^{2} F}{\partial x^{m} \partial y^{k}} y^{m}-\frac{\partial F}{\partial x^{k}}=0 \\
& \Longrightarrow \frac{\partial^{2}(\bar{F}+\beta)}{\partial x^{m} \partial y^{k}} y^{m}-\frac{\partial(\bar{F}+\beta)}{\partial x^{k}}=0 \\
& \Longrightarrow \frac{\partial^{2} \bar{F}}{\partial x^{m} \partial y^{k}} y^{m}-\frac{\partial \bar{F}}{\partial x^{k}}+\frac{\partial^{2} \beta}{\partial x^{m} \partial y^{k}} y^{m}-\frac{\partial \beta}{\partial x^{k}}=0 \\
& \Longrightarrow \frac{\partial^{2} \bar{F}}{\partial x^{m} \partial y^{k}} y^{m}-\frac{\partial \bar{F}}{\partial x^{k}}=0(\text { as } \beta \text { is closed }) \\
& \Longrightarrow \bar{F} \text { is projectively flat. }
\end{aligned}
$$


Conversely, suppose that $\bar{F}$ is projectively flat. Since $\bar{F}$ is projectively flat, therefore $\bar{F}$ will satisfy Hamel's equation

$$
\begin{aligned}
& \frac{\partial^{2} \bar{F}}{\partial x^{m} \partial y^{k}} y^{m}-\frac{\partial \bar{F}}{\partial x^{k}}=0 \\
& \Longrightarrow \frac{\partial^{2}(F-\beta)}{\partial x^{m} \partial y^{k}} y^{m}-\frac{\partial(F-\beta)}{\partial x^{k}}=0 \\
& \Longrightarrow \frac{\partial^{2} F}{\partial x^{m} \partial y^{k}} y^{m}-\frac{\partial F}{\partial x^{k}}-\frac{\partial^{2} \beta}{\partial x^{m} \partial y^{k}} y^{m}+\frac{\partial \beta}{\partial x^{k}}=0 \\
& \Longrightarrow \frac{\partial^{2} F}{\partial x^{m} \partial y^{k}} y^{m}-\frac{\partial F}{\partial x^{k}}=0(\text { as } \beta \text { is closed }) \\
& \Longrightarrow F \text { is projectively flat. }
\end{aligned}
$$

Theorem 3.3. Let $(M, F)$ be a Finsler space with first approximate Matsumoto metric. If $F$ is projectively flat, then it is with reversible geodesics.

Proof. Applying Hammel's equations, one can easily see that $F$ is projectively flat if and only if $\bar{F}$ is projectively flat, which implies that both $F$ and $\vec{F}$ are projectively equivalent to the standard Euclidean metric therefore F must be projective to $\bar{F}$, hence by Lemma 2.6, F must be with reversible geodesics.

Remark 3.4. The converse of the Theorem 3.3 is not true. It can be easily verified with metrics constructed in $([9,10])$.

\section{Finsler metrics associated with weighted quasi-metrics}

It is well known fact that the Riemannian space are expressible as metric spaces. If $(M, \alpha)$ is a Riemannian space, then one can define the induced metric space $\left(M, d_{\alpha}\right)$ with the metric $d_{\alpha}: M \times M \rightarrow[0, \infty)$

$$
\mathrm{d}_{\alpha}(x, y)=\inf _{\gamma \in \Gamma_{x y}} \int_{a}^{b} \alpha(\gamma(t), \dot{\gamma}(t)) d t
$$

where $\Gamma_{x y}=\{\gamma:[a, b] \rightarrow M$ such that $\gamma$ is a piecewise smooth with $\gamma(a)=x, \gamma(b)=y\}$ is the set of curves joining $\mathrm{x}$ and $\mathrm{y}, \dot{\gamma}$ is the tangent vector to $\gamma$ at $\gamma(\mathrm{t})$.

One can easily verify that $d_{\alpha}$ is a metric on M. One knows $([7,10])$ that Finsler structures are more general structures than Riemannian ones. Similar to the Riemannian case, one can induce a metric $d_{F}$ on a Finsler space $(M, F)$, defined by $d_{F}: M \times M \rightarrow[0, \infty)$

$$
d_{F}(x, y)=\inf _{\gamma \in \Gamma_{x y}} \int_{a}^{b} F(\gamma(t), \dot{\gamma}(t)) d t
$$

However, in this case, unlike the Riemannian case, $d_{F}$ lacks the symmetry condition, i.e, $d_{F}(x, y) \neq d_{F}(y, x)$ in general. In fact, $d_{F}$ is a special case of quasi-metric. Recall [3] that a quasi-metric $d$ on a set $X$ is a function $d: X \times X \rightarrow[0, \infty)$ satisfying the following conditions:

(i) Positiveness: $d(x, y)>0$ if $x \neq y, d(x, x)=0$;

(ii) Triangle inequality: $d(x, y) \leqslant d(x, z)+d(z, y)$;

(iii) Separation axioms: $d(x, y)=d(y, x)=0 \Longrightarrow x=y$,

for any $x, y, z \in X$.

There is an special class of quasi-metric spaces, known as weighted quasi-metric spaces $(M, d, \omega)$, where $d$ is a quasi-metric on $M$ for which there exist a function $\omega: M \rightarrow[0, \infty)$, called the weight of $d$, that satisfies 
(iv) Weightability: $d(x, y)+w(x)=d(y, x)+w(y), \forall x, y \in M$.

In the case of weighted function $\omega$ is real valued, $\omega$ is called generalized weight.

It is to be remarked that if $(M, d)$ is a metric space, then it can be regarded as weighted metric space with a weighted function $\omega=$ constant. Next, we have the following theorem:

Theorem 4.1. Let $(M, F)$ be $n$-dimensional simply connected smooth Finsler manifold with $F$ as first approximate Matsumoto metric. Then, F induces generalized weighted quasi-distance $\mathrm{d}_{\mathrm{F}}$ on $M$.

Proof. We consider that $(M, F)$ is the Finsler space with $F=\alpha+\beta+\frac{\beta^{2}}{\alpha}$, which can be written as $F=\bar{F}+\beta$, where $\bar{F}=\alpha+\frac{\beta^{2}}{\alpha}$ is an absolute homogeneous Finsler metric on $M$ and $\beta$ an exact 1 -form.

Let $\gamma_{x y} \in \Gamma_{x y}$ be an F-geodesic, which is in the same time $\bar{F}$-geodesic, then from Equation (4.2), we get

$$
\begin{aligned}
d_{F}(x, y) & =\int_{a}^{b} F(\gamma(t), \dot{\gamma}(t)) d t \\
& =\int_{a}^{b}\left(\alpha+\beta+\frac{\beta^{2}}{\alpha}\right) d t \\
& =\int_{a}^{b}\left(\alpha+\frac{\beta^{2}}{\alpha}\right) d t+\int_{\gamma_{x y}} \beta \\
& =\int_{\gamma_{x y}} \alpha+\frac{\beta^{2}}{\alpha}+\int_{\gamma_{x y}} \beta .
\end{aligned}
$$

Consider a fixed point $a \in M$ and define the function $\omega_{a}: M \rightarrow R$ by $\omega_{a}(x):=d_{F}(a, x)-d_{F}(x, a)$.

From the Equation (4.3) it follows that

$$
\omega_{\mathrm{a}}(x)=\int_{\gamma_{\mathrm{a} x}} \beta-\int_{\gamma_{x \mathrm{a}}} \beta=-2 \int_{\gamma_{\mathrm{xa}}} \beta,
$$

where we have used the Stokes theorem for the 1-form $\beta$ on the closed domain $\mathrm{D}$ with boundary $\partial \mathrm{D}:=$ $\gamma_{a x} \cup \gamma_{x a}$.

It can be easily seen that $\omega_{a}$ is an anti-derivative of $\beta$. This is well defined if and only if the integral in the R.H.S. of Equation (4.4) is path independent, i.e., $\beta$ must be exact.

Then $d_{F}$ is a weighted quasi-metric with generalized weight $\omega_{a}$. Next we have

$$
\begin{aligned}
d_{F}(x, y)+\omega_{a}(x) & =\int_{\gamma_{x y}} \alpha+\frac{\beta^{2}}{\alpha}+\int_{\gamma_{x y}} \beta+\int_{\gamma_{a x}} \beta-\int_{\gamma_{x a}} \beta \\
& =\int_{\gamma_{x y}} \alpha+\frac{\beta^{2}}{\alpha}-\int_{\gamma_{x a}} \beta-\int_{\gamma_{y a}} \beta
\end{aligned}
$$

where we have again used Stokes theorem for the 1-form $\beta$ on the closed domain with boundary $\gamma_{a x} \cup$ $\gamma_{x y} \cup \gamma_{y a}$. Similarly, we can find

$$
d_{F}(y, x)+\omega_{a}(y)=\int_{\gamma_{y x}} \alpha+\frac{\beta^{2}}{\alpha}-\int_{\gamma_{y a}} \beta-\int_{\gamma_{x a}} \beta,
$$

and hence $d_{F}$ is a weighted quasi-metric with generalized weight $\omega_{a}$.

Recall [3] the following lemma for later use. 
Lemma 4.2. Let $(\mathrm{M}, \mathrm{d})$ be any quasi-metric space. Then $\mathrm{d}$ is weightable if and only if there exist a function $\omega: M \rightarrow[0, \infty)$ such that

$$
d(x, y)=f(x, y)+\frac{1}{2}[w(y)-w(x)], \forall x, y \in M,
$$

where $\mathrm{f}$ is the symmetrized distance of $\mathrm{d}$. Moreover, we have

$$
\frac{1}{2}|\omega(x)-\omega(y)| \leqslant f(x, y), \forall x, y \in M .
$$

At this juncture, it is to be remarked that if $(M, F)$ is a Finsler space with $F$ as a first approximate Matsumoto metric, then the induced quasi-metric $d_{F}$ and the symmetrized metric $f$ induce the same topology on $M$ (for proof see lemma 4 of [3]). Further, from the Lemma 4.2 it can be seen that the assumption of $\omega$ to be smooth is not essential. In fact, if $d_{F}$ is a weighted quasi-metric, the function $\omega$ is differentiable everywhere on $M$. Therefore, 1 -form $\beta$-exists almost everywhere on $M$.

Next, we discuss an important geometric property concerning the geodesic triangles.

Theorem 4.3. If $(\mathrm{M}, \mathrm{F})$ is a Finsler space with first approximate Matsumoto metric, then the perimeter of any geodesic triangle on $M$ dose not depend on the orientation, i.e.,

$$
d_{F}(x, y)+d_{F}(y, z)+d_{F}(z, x)=d_{F}(x, z)+d_{F}(z, y)+d_{F}(y, x)
$$

$\forall x, y, z \in M$.

Proof. Since the first approximate Matsumoto metric $F=\alpha+\beta+\frac{\beta^{2}}{\alpha}$ can be treated as the Randers change of absolute homogeneous Finsler metric $\bar{F}=\alpha+\frac{\beta^{2}}{\alpha}$, i.e., $F=\bar{F}+\beta$ with $d \beta=0$, from Theorem 4.1, it follows that the quasi-metric is weightable and therefore equation (4.5) holds good. By using the formula (4.5), an elementary computation gives the required result (4.6).

\section{References}

[1] M. Crampin, Randers spaces with reversible geodesics, Publ. Math. Debrecen, 67 (2005), 401-409. 1, 2, 2

[2] G. Hamel, Über die Geomtrieen in denen die Geraden die Kürzeinten sind, Math. Ann., 57 (1903), 231-264. 3

[3] H. P. A. Kunzi, V. Vajner, Weighted quasi-metrics, Annals New York Acad. Sci., 728 (1994), 64-77. 4, 4, 4

[4] I. Masca, S. V. Sabau, H. Shimada, Reversible geodesics for ( $\alpha, \beta)$-metrics, Intl. J. Math., 21 (2010), 1071-1094. 1, 2, 2

[5] I. Masca, S. V. Sabau, H. Shimada, Two dimensional $(\alpha, \beta)$-metrics with reversible geodesics, Publ. Math. Debrecen, 82 (2013), 485-501. 1

[6] S. V. Sabau, H. Shimada, Finsler manifolds with reversible geodesics, Rev. Roumaine Math. Pures Appl., 57 (2012), 91-103. 1, 2, 2.5, 2.6, 3, 3, 3

[7] Z. Shen, Differential geometry of sprays Finsler spaces, Kluwer Academic Publishers, Dordrecht, (2001). 2, 4

[8] Z. Shen, Lectures in Finsler geometry, World Scientific, USA, (2001).

[9] Z. Shen, On projectively flat ( $\alpha, \beta)$-metrics, Canad. Math. Bull., 52 (2009), 132-144. 3.4

[10] Z. Shen, G. C. Yiedrin, On a class of projectively flat metrics with constant flag curvature, Canad. J. Math., 60 (2008), 443-456. 3.4, 4

[11] P. Vitolo, A representation theorem for quasi-metric space, Topology Appl., 65 (1995), 101-104. 